\title{
Circadian clock genes are differentially modulated during the daily cycles and chronological age in the social honeybee (Apis mellifera)
}

\author{
Fabiano C. P. Abreu ${ }^{1}$, Flávia C. P. Freitas ${ }^{1}$, Zilá L. P. SimÕes ${ }^{2}$ \\ ${ }^{1}$ Departamento de Genética, Faculdade de Medicina de Ribeirão Preto, Universidade de São Paulo, Ribeirão Preto, Brazil \\ ${ }^{2}$ Departamento de Biologia, Faculdade de Filosofia, Ciências e Letras de Ribeirão Preto, Universidade de São Paulo, \\ Ribeirão Preto, Brazil
}

Received 19 July 2017 - Revised 9 November 2017 - Accepted 6 December 2017

\begin{abstract}
The circadian clock is an advantageous adaptive system that enables organisms to predict and anticipate the daily environmental changes. The circadian rhythms are generated molecularly through the expression of clock genes, based on autoregulatory feedback loops. Honeybees are an excellent model to investigate how the circadian rhythms are modulated accordingly to the social context, behavioral plasticity, and task-related activities. Here, we show how the clock genes behave during the daily cycles in adult worker heads of Apis mellifera. Our results point to the clock genes period and cryptochrome as essential regulators of the circadian rhythms associated to the behavioral maturation in this social insect. We also identified putative miRNA-target and protein-protein interactions involving honeybee clock genes, indicating regulatory networks behind the adjustment of the molecular clock.
\end{abstract}

circadian clock / clock genes / circadian rhythms / honeybees / miRNAs

\section{INTRODUCTION}

Temporal coordination of diverse physiological and behavioral processes allows organisms to adapt to environmental daily cycles. Internal clocks behind the temporal coordination process orchestrate the expression of specific genes and generate circadian rhythms, resulting in cyclic biological activities around the 24-h period (Dunlap et al. 2004; Bell-Pedersen et al. 2005). The molecular clockwork is highly conserved throughout evolution, and the ongoing

Electronic supplementary material The online version of this article (https://doi.org/10.1007/s13592-017-0558-7) contains supplementary material, which is available to authorized users.

Corresponding author: F. Abreu, fabiano.genetica@usp.br Manuscript editor: Klaus Hartfelder identification of their molecular components reveals remarkable similarities and differences between vertebrates, invertebrates, and plants (Reppert and Weaver 2000; Young and Kay 2001; Vansteensel et al. 2008).

Circadian rhythms in insects are well known and a wide range of studies demonstrated their intimate relationship with physiological processes as eclosion, ovoposition, ecdysis, metamorphosis, locomotor activity, behavior, and mating (reviewed by Saunders et al. 2002). Comparative analysis within insects from different orders revealed differences in the neuroanatomy of the circadian clock, genetic components, and modes of regulation of the circadian rhythms (Sandrelli et al. 2008; Tomioka and Matsumoto 2010). Interestingly, the molecular clock of honeybees and ants is more similar to mammals than to Drosophila melanogaster (Rubin et al. 2006; Weinstock et al. 2006; Ingram et al. 2012; Sadd et al. 2015). The honeybee cryptochrome (cry-m), for 
example, is an ortholog to its correspondent in mammals and is absent in Drosophila genome (Rubin et al. 2006).

The molecular studies of the clockwork genes on D. melanogaster and Mus musculus (reviewed by Panda et al. 2002) and their expression pattern (Rubin et al. 2006) were the references for the construction of the theoretical knowledge on clockwork genes of Apis mellifera. It consists in autoregulatory feedback loops caused through the oscillatory expression of canonical clock genes in groups of neurons in the central nervous system (Bloch et al. 2003; Rubin et al. 2006; Fuchikawa et al. 2017). Molecular and phylogenetic analyses identified eight clock genes in Apis mellifera: period (per), cryptochrome (cry-m or mammalian-type cry), clock (clk), cycle $($ cyc), timeout 2 (tim2), par domain protein 1 (pdpl), vrille (vri), and clockwork orange (cwo) (Weinstock et al. 2006; Rubin et al. 2006; Rodriguez-Zas et al. 2012). The autoregulatory cycle starts with the formation of the heterodimer CLK/CYC that binds to regulatory sequences and activates the expression of per and cry-m. The products of per and $c r y-m$ accumulate at night in the cytoplasm of oscillatory neurons and act as negative elements forming the heterodimer PER/ CRY-m that enters the nucleus and prevents their own expression binding to CLK/CYC heterodimer (Rubin et al. 2006). The functional domains of VRI and PDP1 are highly conserved to D. melanogaster domains. In honeybees, clk and cyc present putative binding sites for both VRI an PDP1, suggesting another autoregulatory feedback loop (Cyran et al. 2003; Rubin et al. 2006; Bloch 2010). The role of cwo and tim2 in the modulation of the circadian rhythms in Hymenoptera is still unknown. Beyond the canonical clock genes, hundreds of transcripts exhibit circadian oscillations around the zeitgeber in brains of nurses and foragers of honeybees reflecting the clockcontrolled genes (Rodriguez-Zas et al. 2012).

The post-transcriptional regulation performed by microRNAs also plays an important role in modulating the oscillatory expression of clock genes (Kojima et al. 2011, Luhur et al. 2013).
MicroRNAs were found to be rhythmic in Drosophila brain, mouse CNS, mouse retina, and Arabidopsis, and some of them are directly involved in modulating the circadian period and response to light (Cheng et al. 2007, Xu et al. 2007, Jung et al. 2007, Yang et al. 2008, Sire et al. 2009, Kadener et al. 2009). In honeybees, a set of microRNAs were identified as involved in caste determination (Weaver et al. 2007; Chen et al. 2010; Ashby et al. 2016), memory and learning (Cristino et al. 2014; Qin et al. 2014), foraging (Behura and Whitfield 2010; Liu et al. 2012a, b), embryogenesis (Pires et al. 2016; Freitas et al. 2017), and reproductive status (Macedo et al. 2016). However, there are no studies showing whether miRNAs affect the circadian system in honeybees.

Due to highly organized society, extraordinary cognitive capacity, and complex behavior, honeybees are an excellent model to investigate how the circadian rhythms can be influenced by the social interactions (Meshi and Bloch 2007; Bloch 2010; Shemesh et al. 2010; Eban-Rothschild et al. 2012; Nagari and Bloch 2012). The social synchronization is as important as light and temperature to entrain the clock of honeybees, and adult workers can exhibit different activity rhythms depending on the colony ambient and contact to conspecifics (Frisch and Koeniger 1994; Nagari and Bloch 2012; Fuchikawa et al. 2016). Further, the behavioral plasticity of honeybees associated to the division of labor is thought to be closely related to modulation of circadian rhythms (Bloch et al. 2001; Shemesh et al. 2007; Shemesh et al. 2010). Here, we tested if the daily oscillation of canonical clock genes changes in workers with different ages by assessing the expression profiles of the genes per, cry-m, clk, cyc, cwo, tim 2, vri, and $p d p 1$ in heads of young (3- and 7-day-old) and old (15- and 25-day-old) bees. We used computational analysis to predict microRNAs as regulators of clock genes and to rebuild a protein interaction network that illustrates the physical interactions between the products of the canonical clock genes and other proteins. Our aim was to show how the circadian rhythm plasticity is related to the 
complex behavior of honeybees through clock gene expression in a special colony, using an Africanized A. mellifera population from a tropical region. Studies focused on the molecular clock of social insects certainly will shed light on how the eusociality could be related to the evolution of internal clocks.

\section{MATERIAL AND METHODS}

\subsection{Bee samples}

We worked with experimental colonies in the "single-cohort colony" model, which allow us the control of the adulthood, behavioral maturation, and sample bees of the same age. We removed honeycomb frames containing pupae, close to eclosion, from source colonies of Africanized honeybees (Apis mellifera) from the experimental apiary of the Department of Genetics at the Faculdade de Medicina de Ribeirão Preto (Ribeirao Preto, Brazil) and transferred them to dark incubator (32 \pm $0.5{ }^{\circ} \mathrm{C}, 55 \pm 5 \%$ relative humidity) to obtain emerging bees with 0-24 h. Approximately 1500 newly emerged worker bees were paintmarked on the thorax and introduced in an experimental observation hive provided with a virgin queen and a frame with pollen, honey, brood, and empty cells where the queen could lay eggs. The observation hive was kept in a dark room (to prevent light stimulation) with constant temperature $\left(28^{\circ} \mathrm{C} \pm 1\right)$. An exit was built in the experimental hive so that the bees could fly away from the hive, collect food (forage), and experience the sunlight. Worker bees with $3,7,15$, and 25 days old were collected at four zeitgeber times $(7,12,17$, 22 h) under red light. Sampled bees $(n=3-5$ per zeitgeber time) were individually immersed in liquid nitrogen and had their entire heads collected for subsequent extraction of total RNA. The sampling procedure was performed from September to October, during the spring. We kept the colony in the absence of light as soon as we started the experiment and did not use an artificial light-dark (LD) regime to synchronize the colony population. However, the colony was susceptible to be socially synchronized by free-flying foragers that experienced the sunlight outside, so as by the nurse bees during the experimental flies.

\subsection{RNA extraction for mRNA expression profile}

Total RNA was extracted from entire heads of individually sampled bees using Trizol reagent (Invitrogen) accordingly to the manufacturer protocol. The extracted RNA was treated with RNAse-free DNAse (DNase I Amplification Grade-Invitrogen), and the total RNA was quantified using the NanoDrop® ND-1000 Spectrophotometer V3.03 (NanoDrop Technologies).

For mRNA expression analysis, an aliquot of $1.5 \mu \mathrm{g}$ of total RNA was reverse transcribed using an oligoDT and the specific enzyme SuperScript $^{\mathrm{TM}}$ II Reverse Transcriptase $(200 \mathrm{U} / \mu \mathrm{L}$, Invitrogen), as described by the manufacturer. Specific oligonucleotides were designed for per, cry-m, clk, cyc , tim 2, cwo , pdp1, and vri using Primer3 tool (http://bioinfo.ut.ee/primer3-0.4.0/) and validated through qPCR standard curve method (Livak and Schmittgen 2001) to evaluate their efficiency. Oligonucleotide sequences are listed in Supplemental Table S1. RT-cDNA samples were used as templates for qPCR amplification of the clock genes with the SYBR® Green Master Mix $2 \times$ (Applied Biosystems) and 7500 Real-time PCR System (Applied Biosystems). The gene expression was normalized against the ribosomal protein L32 (rpl32), as suggested by Lourenço et al. (2008) and calculated according to the $2^{-}$ $\Delta \Delta \mathrm{Ct}$ method (Livak and Schmittgen 2001). Statistical analyses were performed using the oneway ANOVA test and Tukey's pairwise comparison $(p<0.05)$.

\subsection{Gene regulatory network for miRNA- mRNA interactions}

We selected a region of $1000 \mathrm{pb}$ downstream to the stop codon of clock genes to represent the 3' UTR and performed the search for miRNA binding sites using the RNAhybrid tool (Kruger and Rehmsmeier 2006). We selected this parameter because the sequences of untranslated regions $\left(5^{\prime}\right.$ and 3' UTRs) are not available for every gene in 
the current version of honeybee genome (The Honeybee Genome Sequencing Consortium 2006; Elsik et al. 2014). To reduce false-positive miRNA-target interactions, we performed the search for miRNA binding sites in 3' UTRs of Drosophila genes and considered for further analysis the miRNA-target interactions conserved in both species. This approach has proven to be efficient in detecting functional miRNA-binding sites in honeybees as demonstrated by publications of our group (Cristino et al. 2014; Freitas et al. 2017). We considered only the interactions that are based on the seed region of miRNAs (2$7 \mathrm{nt})$, free energy $\leq-20 \mathrm{Kcal} / \mathrm{mol}$, and $p$ value $<$ 0.05 .

\subsection{Protein-protein interaction network}

To build a putative protein-protein interaction (PPi) network, we retrieved proteins that interact with clock genes in Drosophila and Mus musculus from BioGrid repository (version 3.4.138) (Chatr-aryamontri et al. 2017). The PPis involving conserved genes in honeybee were used to build a PPi network using Cytoscape tool, version 3.4.0 (Shannon et al. 2003). Functional analysis of PPi network was carried out based on information available at GeneOntology Consortium (www.geneontology.org), PANTHER Classification System (www.phanterdb.org) $(p \leq 0.05$ ), and FlyBase (www.flybase.org).

\section{RESULTS}

\subsection{Chronological and daily patterns of clock gene expression during the adult development of $A$. mellifera}

Our quantitative PCR (qPCR) analysis revealed that the clock genes per, cry-m, clk, $c y c$, tim 2, pdp1, vri, and cwo are expressed in heads of adult workers in the chronological ages of $A$. mellifera (Figure 1). The clock genes per, cry- $m$, and $c$ wo exhibited a similar expression pattern showing higher transcriptional levels in heads of old bees (25-dayold) compared to younger bees. Conversely, the clock gene $p d p 1$ showed an opposite expression pattern, with higher transcriptional levels in the heads of young bees (3-day-old) compared to older bees. The average transcriptional levels of $c l k$ were higher in heads of 25day-old bees compared to 3- and 15-day-old bees, but showed no significant differences related to 7-day-old bees. For $c y c$ and vri, 15-day-old bees showed differences in their average transcriptional levels compared to 3day-old and 7-day-old bees, respectively. The average transcriptional levels of tim2 did not vary with different ages.

Daily expression patterns of clock genes in heads are evident in at least one chronological age. Based on the amplitude of the expression profile variation, we classified the transcriptional oscillations as absent, present, or attenuated present (Figures 2 and 3). Transcriptional oscillations of per and cry- $m$ within a day were absent or attenuated present in young bees (3- and 7-dayold) and showed high amplitude in older bees (15and 25-day-old). Both per and cry-m presented peaks of expression at the beginning of the day ( $7 \mathrm{~h})$ and in the middle of the dark phase $(22 \mathrm{~h})$ in the heads of 15-day-old bees. In 25-day-old bees, we observed a peak of expression of per and cry 2 in the dark phase (22 h). For $c l k$, we found a clear transcriptional oscillation in heads of 25-day-old bees with one peak at the light phase $(12 \mathrm{~h})$ and another at the dark phase (22h) (Figure 2 (b)). For $c y c$, the transcriptional oscillation was more attenuated in the young bees and similar to $c l k$ oscillation in 25-day-old bees.

Interestingly, $p d p 1$ showed a strong daily oscillation in heads of young bees (3-dayold) compared to vri, while the opposite was observed for vri in old bees (25-dayold) (Figure 3 (a)). Both $p d p 1$ and vri showed peaks of expression in the light phase of the day $(12 \mathrm{~h})$. For $c w o$, we observed transcriptional oscillations in heads of 3-, 15-, and 25-day-old bees (Figure 3 (c)). A peak of cwo expression was observed at the light phase $(17 \mathrm{~h})$ in heads of 3-day-old and 15-day-old bees, what implies a circadian oscillation at these ages. In 25-day-old bees, the levels of cwo are high in the light phase $(17 \mathrm{~h})$ and reach a peak of expression at the dark phase $(22 \mathrm{~h})$. Transcriptional oscillation of tim2 was absent within a day 


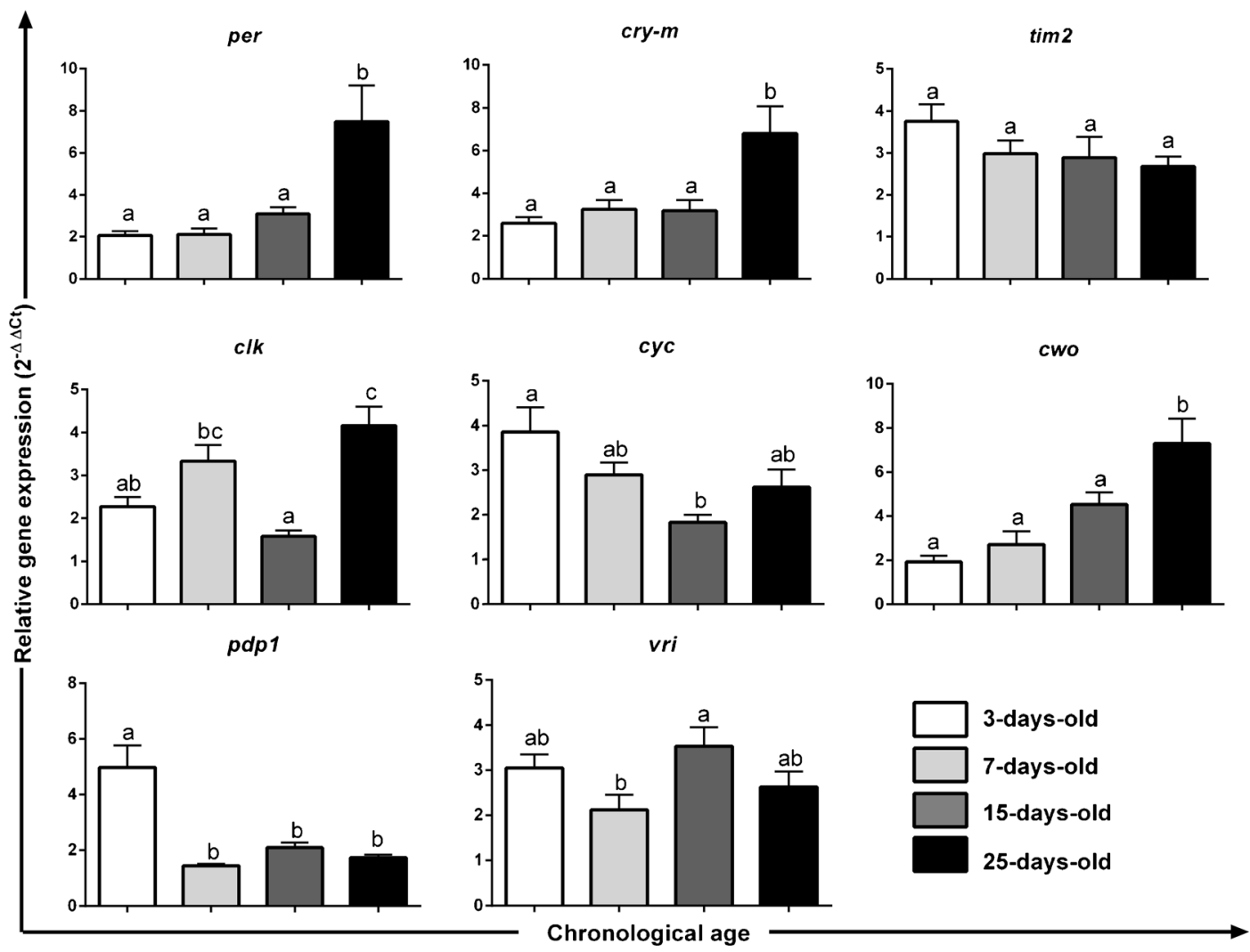

Figure 1. Transcriptional levels of clock genes during the chronological age in worker heads of A. mellifera . qPCR was used to obtain the relative gene expression which was normalized to the endogenous gene rpl32 through the $2^{-\Delta \Delta \mathrm{Ct}}$ method. For each adult age, we used the mean expression of clock genes in heads of three to five individuals calculated from each time point collected during the day. Statistical analysis was performed using one-way ANOVA, followed by Tukey's pairwise comparison, $p<0.05$.

during the chronological age of worker bees (Figure 3 (b)).

\subsection{Interaction networks}

Our computational analysis revealed that the clock genes per, cry-m, clk, cyc, tim2, pdp1, $v r i$, and $c w o$ exhibited putative binding sites in their 3' UTR that are supposed to interact with miRNAs in A. mellifera. Due to the high number of miRNA-mRNA interactions recovered by our analysis and taking into account that computational predictions for binding sites of miRNAs can generate false-positive results, we selected miRNAs previously listed in other insects and with a possible role in regulating circadian and neuronal functions at least in D. melanogaster. We reconstructed a miRNA-mRNA network using interactions predicted in both species (Figure $4 \mathrm{a}$ ). The results point to potential miRNAs to regulate the clock genes that are well studied in fruit flies or reported to be involved in honeybee developmental processes, like let-7, miR-8, miR$9 a, m i R-34, m i R-124$, and bantam.

We found physical interactions for the clock genes per, cry-m, clk, cyc, tim2, pdp1, and vri based on curated data for orthologs in D. melanogaster and M. musculus. Based in our protein-protein interaction network (Figure 4b), we searched for gene ontology $(p \leq 0.05)$ (Supplemental Figure S1) and found biological processes linked to cellular, metabolic, and 


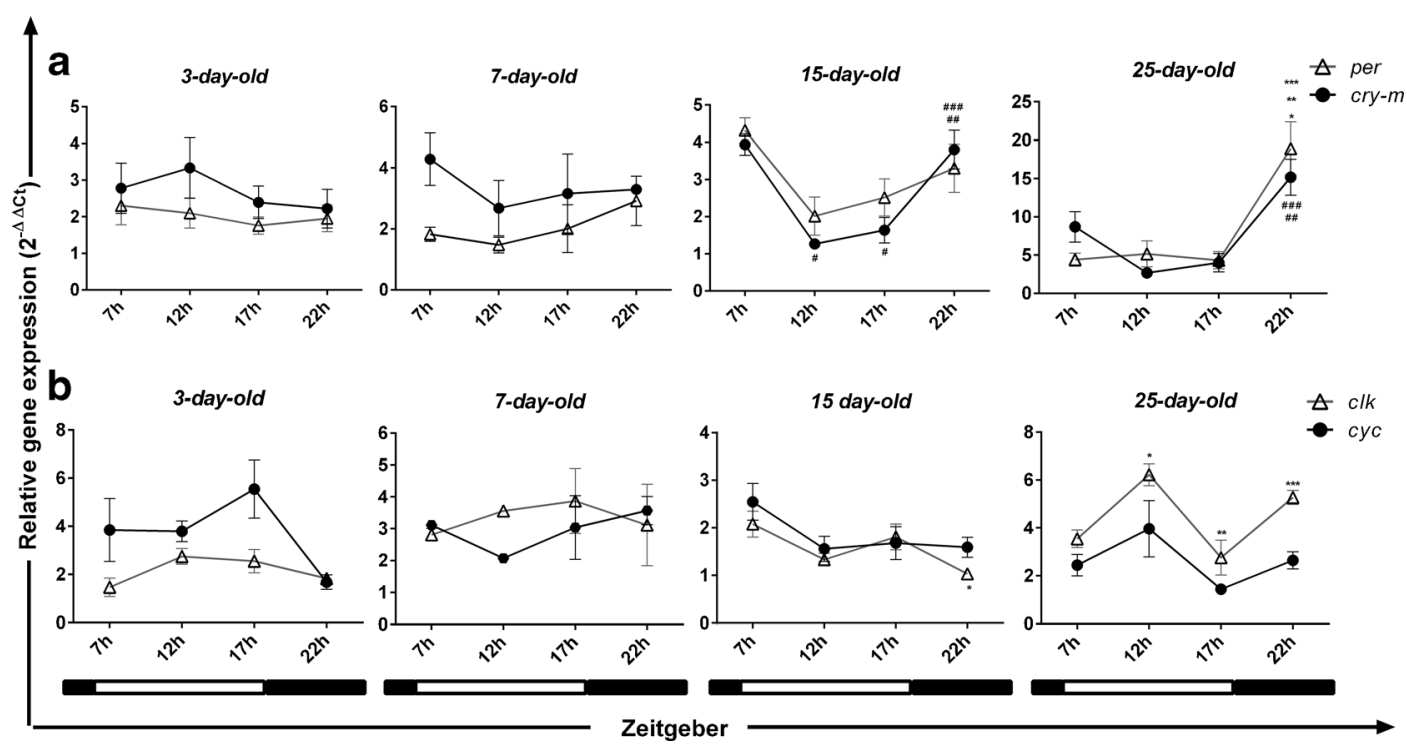

Figure 2. Daily expression patterns of clock genes per and cry-m $(a)$ and $c l k$ and $c y c(b)$, involved in a first autoregulatory feedback loop, during the chronological age in worker heads of A. mellifera. qPCR was used to obtain the relative gene expression which was normalized to the endogenous gene rpl32 through the $2^{-\Delta \Delta \mathrm{Ct}}$ method. Each zeitgeber time represents the mean expression in heads of three to five individuals. Statistical analysis was performed using one-way ANOVA, followed by Tukey's pairwise comparison, $p<0.05$. * or ${ }^{\#}$ different from $7 \mathrm{~h}, * *$ or ${ }^{\# \#}$ different from $12 \mathrm{~h}, * * *$ or ${ }^{\# \# \#}$ different from $17 \mathrm{~h}$. Black and white bars at the bottom reflect the sunlight hours with dark and light daily phases, respectively.

developmental regulation, mainly related to enzymatic, catalytic, and structural activities.

\section{DISCUSSION}

In this work, we analyzed the chronological and daily patterns of clock gene expression in heads of A. mellifera using four different adult ages representing young (3- and 7-day-old) and old (15- and 25-day-old) bees living in a singlecohort colony. The characteristics of a singlecohort colony as same-aged bees made possible to test the expression of clock genes are influenced by the chronological age and/or behavior. Our analysis showed that the average daily expression of per is higher in heads of old bees (25day-old) compared to young bees (3- and 7-dayold) (Figure 1), consistent with Toma et al. (2000). In addition, our analysis pointed to higher daily expression of per in heads of 25-day-old bees compared to 15-day-old bees. We observed these same features for $c r y-m$ and $c w o$, which have not been demonstrated before. The higher transcriptional levels of per, cry-m, and $c w o$ in heads of 25-day-old bees may be related to the intense forager activity that depends on strong circadian rhythmicity. A previous study did not observe differences in the expression level of per in brains of young bees (8- to10-day-old) compared to old bees (23- to 25-day-old) using singlecohort colonies (Bloch et al. 2001). Although it seems contradictory, other findings support that expression levels of per transcripts in the central nervous system of worker bees are not strictly linked to their chronological age but depend on other factors such as precocious foraging, a characteristic of both single-cohort colonies reared in field or laboratory (Toma et al. 2000; Bloch et al. 2001). In our analysis, average daily expression of per, cry- $m$, and $c w o$ in heads of young bees (3-7day-old) compared to potential foragers (15-dayold) showed no difference (Figure 1). The absence of foragers in the initial population of our singlecohort colony (composed of newly emerged workers) likely affects per, cry-m, and cwo mRNA levels, and the expression of these genes 


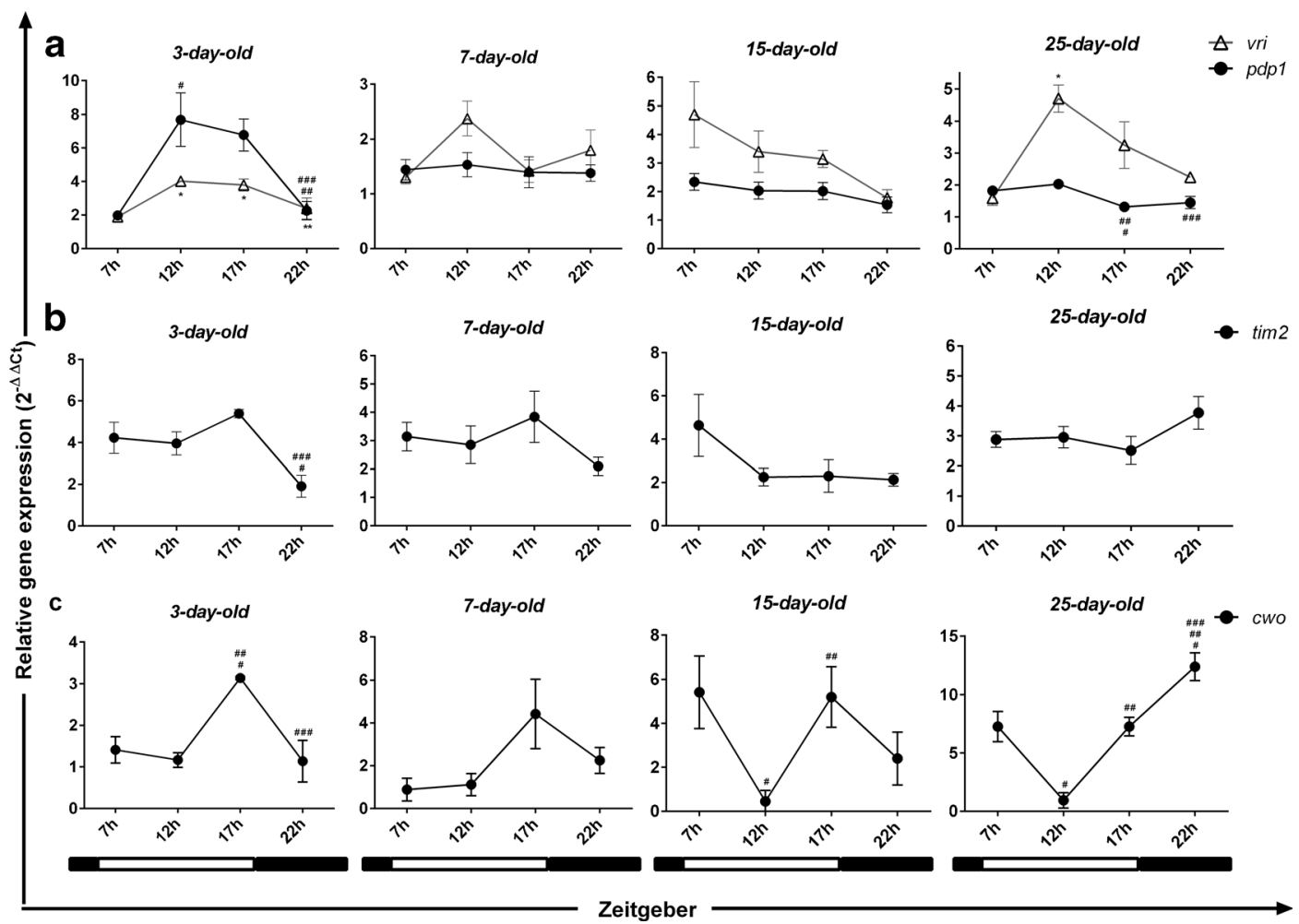

Figure 3. Daily expression patterns of clock genes pdpl and vri (a), tim2 (b), and cwo (c) during the chronological age in worker heads of $A$. mellifera. qPCR was used to obtain the relative gene expression which was normalized to the endogenous gene $r p l 32$ through the $2^{-\Delta \Delta C t}$ method. Each zeitgeber time represents the mean expression in heads of three to five individuals. Statistical analysis was performed using one-way ANOVA, followed by Tukey's pairwise comparison, $p<0.05$. * or ${ }^{\#}$ different from $7 \mathrm{~h}$, ** or ${ }^{\# \#}$ different from $12 \mathrm{~h}$, *** or ${ }^{\# \# \#}$ different from $17 \mathrm{~h}$. Black and white bars at the bottom reflect the sunlight hours with dark and light daily phases, respectively.

reaches higher levels earlier in adult life as a signal to prepare young bees to forage. We did not find variation in the average levels of per, $c r y-m$, and cwo comparing 15-day-old bees and 3-7-day-old bees; nonetheless, we observed a striking difference between their daily transcriptional fluctuation in heads at least for per and cry-m (Figure 2 (a)). An absence or attenuated oscillation in daily expression of both genes found in young bees compared to the strong oscillation in old bees is consistent with other studies (Bloch et al. 2001; Shemesh et al. 2007). Our results suggest that the expression levels of per and $c r y-m$ are associated with the behavioral plasticity in honeybees and that the oscillations in their expression reflect the division of labor between young and old bees. While young bees (nurses) work arrhythmically around the clock inside the colony in brood-care activities, old bees (foragers) need to be strongly rhythmic to develop complex tasks as foraging, sun navigation, learning, memory, and dance language. In contrast, Fuchikawa et al. (2017) found that the oscillation on protein levels of per is similar in brains of behaviorally rhythmic foragers and arrhythmic nurses. This observation in soma with our analysis suggests that cycling on proteins levels does not require cycling of the transcripts in arrhythmic nurses. Taken together, our analysis supports the idea that the ontogeny of circadian rhythms is closely linked to the division of labor in social insects and transcriptional levels of per and cry-m. Our results point to the clock genes per and cry-m as candidate molecular markers of behavioral maturation in honeybees. 

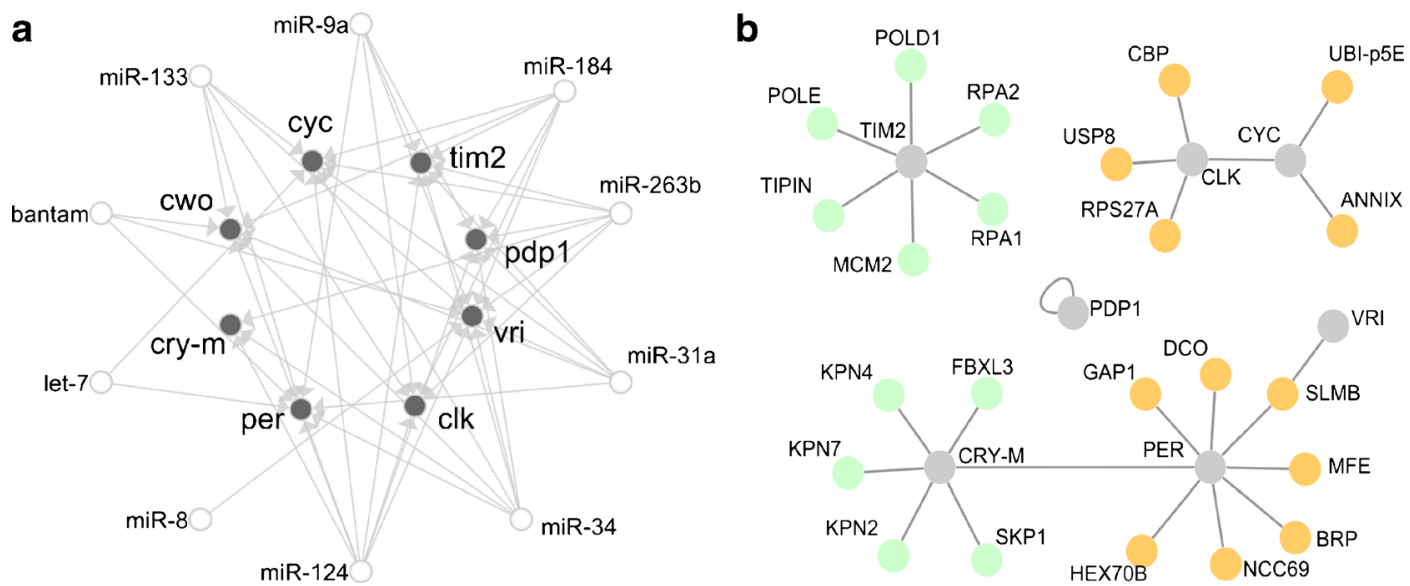

Figure 4. a miRNA-mRNA interaction network. The network illustrates the putative miRNAs that were predicted to bind to 3' UTRs in transcripts of clock genes in the genome of A. mellifera. The prediction was based on previously determined parameters $(\Delta G \leq-20 \mathrm{Kcal} / \mathrm{mol} ; p$ value $<0.05)$. White nodes and gray nodes represent the predicted miRNAs and the target clock genes, respectively. b Protein-protein interaction network representing the putative physical interactions between clock genes and other proteins in A. mellifera. The analysis was based on curated data available for D. melanogaster and M. musculus in the BioGRID online tool (http://thebiogrid.org/). Physical interactions based on orthology with $D$. melanogaster correspond to orange nodes linked to clock genes per, cyc, clk, and vri (gray nodes). Interactions based on orthology with M. musculus represent the green nodes linked to clock genes $c r y$ - $m$ and tim2 (gray nodes).

Noteworthy, expression levels of $c l k$ do not oscillate within a day in young bees (3- or 7day-old) but it does oscillate in old bees (25-dayold). Our data contrasts to a previous study that reported absence of oscillation in $c l k$ in forager bees (Rubin et al. 2006). The daily transcriptional levels of $c y c$ are synchronized with $c l k$ in 25-dayold bees and it is unlikely that the moderate oscillations observed are due to the circadian influence. In mammals, tim 2 is expressed in retinoid cells, which indicates that this gene is related to photoreceptor function (Sangoram et al. 1998; Takumi et al. 1999). In another Hymenoptera, Ceratosolen solmsi, tim2 has an arrhythmical expression in males that live inside the syconium of flowers in the absence of light. In young females that leave the syconium earlier, tim 2 showed a rhythmic expression around the day, suggesting that the oscillation of this gene is light dependent ( $\mathrm{Gu}$ et al. 2014). However, our data show that tim 2 did not fluctuate during all the chronological age of worker bees (Figure 3 (b)). This result is in accordance with those reported by Rubin et al. (2006). Further experiments are necessary to address how tim 2 participates in the circadian mechanism in honeybees.

Here, we showed for the first time the expression profiles of vri and $p d p 1$ in heads of honeybees. Besides the classical role of $p d p 1$ and vri in the modulation of circadian rhythms, noncircadian roles were found for $p d p 1$ and vri in embryos and larval development of flies (George and Terracol 1997; Reddy et al. 2000; Szuplewski et al. 2003). The strong transcriptional fluctuations detected for $p d p 1$ during the beginning of adult life and later in 25-day-old bees for vri point to different roles during the development of adult workers (Figure 3 (a)). The high transcriptional levels of $p d p 1$ in young bees (3-day-old) may be related to their nutritional status, once it was reported that this gene is affected by the availability of food during larval development in fruit flies (Reddy et al. 2006). As reported in the literature, the diet and feeding regime regulate the internal clock of organisms and we suggest that the amount and differential uptake of nutrients during the chronological age of honeybees affect directly pdp1 expression (Chaudhari et al. 2017). 
Analysis pointed to the conservation of protein domains between Hymenoptera cwo and its ortholog in D. melanogaster, indicating that this gene has conserved the same function within insects (Kadener et al. 2007; Lim et al. 2007; Matsumoto et al. 2007; Ingram et al. 2012; Rodriguez-Zas et al. 2012). We observed that the high transcriptional levels of $c w o$ at $17 \mathrm{~h}$ in heads of old bees (Figure 3 (c)) are similar to those observed by Rodriguez-Zas et al. (2012). Similar fluctuation patterns of $c w o$ expression profile from 7 to $17 \mathrm{~h}$ were observed in heads of 15 day-old and 25-day-old bees, but not at $22 \mathrm{~h}$. We cannot discard that in a social environment, not all the bees will present the same circadian phase, at least when gene expression is considered. In a social community, the members behave differently due to the opportunity to interact each other. The same is supposed to occur with the circadian rhythm. An example is the expression of $c$ wo that demonstrates sensitivity to colony social organization and varies when individual tests were performed. This enables us to say that a single-cohort colony affected the transcriptional modulation since it has a peculiar demographic distribution, at least at the initial experimental phase.

The identification of miRNAs in circadian neurons of $D$. melanogaster suggested that these small non-coding RNAs might regulate the stability or the translation of clk, vri, and cwo (Kadener et al. 2009). Additionally, computational simulations have demonstrated that miRNAs are involved in the modulation of the circadian rhythms altering its amplitude and periodicity (Nandi et al. 2009). Kadener et al. (2009) also described several miRNAs upregulated in circadian neurons of $D$. melanogaster when the enzyme Dicer was disrupted by the knockdown. Some conserved miRNAs found in our analysis are linked to neuronal functions: let-7, $m i R-8, m i R$ $9 a, m i R-34, m i R-124$, and bantam (reviewed by Asgari 2013). Overexpression of bantam, a potential regulator of $c l k$, in adult neurons of fruit flies leads to longer rhythmic periods (Kadener et al. 2009). Overexpression or deletion of let-7 affects the length of circadian rhythms (Chen et al. 2014). It is suggested that let-7 forms a regulatory cycle in the circadian clock being activated indirectly by $\mathrm{CLK} / \mathrm{CYC}$ through the ecdysteroid pathway and negatively regulates the expression of $c w o$. Further, let-7, $m i R-8$, and $m i R-34$ are essential players in preventing neurodegeneration process and aging in D. melanogaster (Liu et al. 2012a, b; Chawla et al. 2016). miR-92a has an oscillatory expression in circadian neurons of fruit flies and modulates the neuronal excitability through regulation of sirt2 (Chen and Rosbash 2016). The miRNAs miR-31a, miR-124, miR$133, m i R-184$, and $m i R-263 b$ (Figure $4 \mathrm{a}$ ) found in our analysis have been detected in the central nervous system of $D$. melanogaster (Kadener et al. 2009), which opens the opportunity to investigate experimentally how these miRNAs are involved in the modulation of circadian rhythms in honeybees.

Beyond the post-transcriptional regulation of clock genes, physical interactions at translational level are important to regulate the stability of clock proteins in the nucleus or in the cytoplasm (Weber et al. 2011; Reischl and Kramer 2011; Crane and Young 2014). Moreover, the canonical clock proteins not only act as transcriptional factors in the circadian machinery but also interact with other products to regulate non-circadian functions. Our protein-protein interaction network (Figure $4 \mathrm{~b}$ ) revealed physical interactions between clock proteins and non-clock proteins related to the biological processes: post-translational regulation, phosphorylation, ubiquitination, and degradation. The discs overgrown (DCO), also called doubletime (DBT), is one of the proteins found to phosphorylate PER contributing to modulate its translational levels in the cytoplasm (Price et al. 1998; Kloss et al. 1998; Cyran et al. 2005; Reischl and Kramer 2011; Weber et al. 2011). The protein supernumerary limb (SLMB) recognizes the phosphorylated site in PER and acts in recruiting other proteins to direct PER to degradation through the ubiquitin-proteasome system (Ko et al. 2002; Chiu et al. 2008). The proteins USP8, RPS27A, and UBI-p5E involved in the ubiquitin-proteasome system interact with CLK and CYC suggesting that a conserved degradation machinery is essential to the clearance of clock gene products. We also observed a physical interaction between VRI and SLMB, suggesting a possible non-circadian role during embryogenesis, as reported in the literature and in a specialized 
database (FlyBase) (George and Terracol 1997; Szuplewski et al. 2010). Another possible role linked to the generation of circadian rhythms was observed for PER and BRP (bruchpilot) interaction; it is known from the literature that BRP is associated with the synaptic circadian plasticity in the visual system of fruit flies (Woźnicka et al. 2015). The brp gene is also described to be involved in functions related to adult locomotor behavior and short-term memory (Wagh et al. 2006; Knapek et al. 2011).

The physical interactions recovered for TIM2 and CRY-m, orthologous in mammals, revealed proteins related to metabolism and DNA replication processes. We reinforce here that all proteins found to interact physically with TIM2 and CRY$\mathrm{m}$ based on M. musculus data present an ortholog in the honeybee, suggesting similar interactions. We observed that all proteins found to interact with TIM2 have an essential role in regulating the chromosome integrity and DNA replication (Gotter et al. 2000; Benna et al. 2010). CRY-m showed two physical interactions with FBXL3 and SKP1, probably originating a complex to polyubiquitinate CRY-m and direct it to degradation. This result suggested a possible role of this complex to regulate CRY-m stability and modulate the circadian rhythms (Xing et al. 2013; Yoo et al. 2013). The physical interactions for CRY-m also revealed proteins involved in the nuclear import, the Karyopherins KPN2, KPN4, and KPN7, as candidates to participate in CRY-m translocation from the cytoplasm to nucleus. Taken together, the reconstruction of protein-protein interaction network allowed us to extend our view on the possible interactions at a translational level that potentially regulate the stability of clock proteins and even establish non-circadian functions along the development of honeybees.

Finally, our work gave us the opportunity to discuss how the circadian clock genes are modulated in the central nervous system of Africanized honeybees reared under special conditions, taking into account that they are localized in a different geographic region compared to the honeybees living in the temperate climate. Here, we added new elements to understand how the conserved circadian machinery, analyzed under these circumstances, so as affected by climatic, geographic, nutritional, and social contexts, modulates the circadian rhythms and the behavioral plasticity in social insects, especially honeybees.

Funding FAPESP, Process No 2014/14194-4.

\section{AUTHORS' CONTRIBUTION}

All authors have contributed equally to the work: Fabiano C.P. Abreu and Zilá L.P. Simões elaborated the idea of this work, experimental procedures were performed by Fabiano C. P. Abreu, and Flavia C.P. Freitas performed the computational analyses.

\section{COMPLIANCE WITH ETHICAL STANDARDS}

Conflict of interest The authors declare that they have no conflict of interest.

Les gènes de l'horloge circadienne sont modulés de façon différentielle au cours des cycles quotidiens et de l'âge chronologique chez l'abeille domestique (Apis mellifera )

horloge circadienne / gene horloge / rythme circadien / abeille / miRNAs

Die Expression von Genen der circadianen Uhr ist differentiell moduliert in Bezug auf die Tageszyklen und das chronologische Alter der sozialen Honigbiene (Apis mellifera)

circadiane Uhr/ Uhr-Gene / circadiane Rhythmen / Honigbiene / micro RNAs

\section{REFERENCES}

Asgari, S. (2013) MicroRNA functions in insects. Insect. Biochem. Mol. Biol. 43 (4): 388-97

Ashby, R., Forêt, S., Searle, I., Maleszka, R. (2016) MicroRNAs in honey bee caste determination. Sci. Rep.. 7; 6: 18794

Behura, S. K., Whitfield, C. W. (2010) Correlated expression patterns of microRNA genes with age-dependent behavioural changes in honeybee. Insect. Mol. Biol. 19: 431-439.

Bell-Pedersen, D., Cassone, V. M., Earnest, D. J., Golden, S. S., Hardin, P. E., Thomas T. L., Zoran, M. J. (2005) 
Circadian rhythms from multiple oscillators: lessons from diverse organisms. Nat. Rev. Genet. 6, 544-56.

Benna, C., Bonaccorsi, S., Wülbeck, C., Helfrich-Förster, C., Gatti, M., Kyriacou, C. P. \& Sandrelli, F. (2010) Drosophila timeless 2 is required for chromosome stability and circadian photoreception. Curr. Biol. 20 (4), 346-352

Bloch, G. (2010) The social clock of the honeybee. J. Biol. Rhythms. 25, 307-17.

Bloch, G., Toma, D. P., Robinson, G. E. (2001) Behavioral Rhythmicity, Age, Division of Labor and period Expression in the Honey Bee Brain. J. Biol. Rhythms. 16 (5), 444-456.

Bloch, G., Solomon, S. M., Robinson, G. E., Fahrbach, S. E. (2003) Patterns of PERIOD and pigment-dispersing hormone immunoreactivity in the brain of the European honeybee (Apis mellifera): age- and time-related plasticity. J. Comp. Neurol. 464 (3): 269-284.

Chatr-aryamontri, A., Oughtred, R., Boucher, L., Rust, J., Chang, C., et al. (2017) The BioGRID interaction database: 2017 update. Nucleic Acids Res. 45(D1):D369-D379.

Chaudhari, A., Gupta, R., Makwana, K., Kondratov, R. (2017) Circadian clocks, diet and aging. Nutr. Healthy Aging. 4, 101-112.

Chawla, G., Deosthale, P., Childress, S., Wu, Y.C., Sokol, N.S. (2016) A let-7-to-miR-125 MicroRNA Switch Regulates Neuronal Integrity and Lifespan in Drosophila. PLoS. Genet. 12 (8): e1006247

Chen, X., Rosbash, M. (2016) MicroRNA-92a is a circadian modulator of neuronal excitability in Drosophila. Nature Commun. 8: 14707.

Chen, X., Yu, X., Cai, Y., Zheng, H., Yu, D., Liu, G., Zhou, Q., Hu, S., Hu, F. (2010) Next-generation small RNA sequencing for microRNAs profiling in the honey bee Apis mellifera. Insect. Mol. Biol. 19: 799-805.

Chen, W., Liu, Z., Li, T., Zhang, R., Xue, Y., Zhong, Y., Bai, W., Zhou, D., Zhao, Z. (2014) Regulation of Drosophila circadian rhythms by miRNA let-7 is mediated by a regulatory cycle. Nat. Commun. 5: 5549.

Cheng, H. Y., Papp, J. W., Varlamova, O., Dziema, H., Russell, B., Curfman, J. P., Nakazawa, T., Shimizu, K., Okamura, H., Impey, S., et al. (2007). microRNA modulation of circadian-clock period and entrainment. Neuron. 54, 813-829.

Chiu, J. C., Vanselow, J. T., Kramer, A., \& Edery, I. (2008). The phospho-occupancy of an atypical SLIMBbinding site on PERIOD that is phosphorylated by DOUBLETIME controls the pace of the clock. Genes Dev. 22 (13), 1758-1772.

Crane, B. R., Young, M. W. (2014). Interactive features of proteins composing eukaryotic circadian clocks. Annu. Rev. Biochem. 83, 191-219.

Cristino, A. S., Barchuk, A. R., Freitas, F. C., Narayanan, R. K., Biergans, S. D., Zhao, Z., Simoes, Z. L., Reinhard, J., Claudianos, C. (2014) Neuroliginassociated microRNA-932 targets actin and regulates memory in the honeybee. Nat. Commun. 20; 5: 5529.
Cyran, S. A., Buchsbaum, A. M., Reddy, K. L., Lin, M. V., Glossop, N. R., Hardin, P. E., Young, M. W., Storti, R. V., Blau, J. (2003) vrille, Pdp1, and dClock form a second feedback loop in the Drosophila circadian clock. Cell 112: 329-341.

Cyran, S. A., Yiannoulos, G., Buchsbaum, A. M., Saez, L., Young, M. W., \& Blau, J. (2005). The double-time protein kinase regulates the subcellular localization of the Drosophila clock protein period. J. Neurosci. 25 (22), 5430-5437.

Dunlap, J., Loros, J., DeCoursey, P. (2004) Chronobiology: biological timekeeping. Sunderland: Sinauer Associates

Eban-Rothschild, A., Shemesh, Y., Bloch, G. (2012) The colony environment, but not direct contact with conspecifics, influences the development of circadian rhythms in honey bees. J. Biol. Rhythms. 27 (3): 217:225.

Elsik, C. G., Worley, K. C., Bennett, A. K., Beye, M., Camara, F., Childers, C. P., ... \& Elhaik, E. (2014). Finding the missing honey bee genes: lessons learned from a genome upgrade. BMC genomics, $15(1), 86$

Freitas, F. C. P., Pires, C. V., Claudianos, C., Cristino, A. S., Simões, Z. L. P. (2017) MicroRNA-34 directly targets pair-rule genes and cytoskeleton component in the honey bee. Sci. Rep. 7: 40884.

Frisch, B., Koeniger, N. (1994) Social synchronization of the activity rhythms of honeybees within a colony. Behav. Ecol. Sociobiol. 35, 91-98

Fuchikawa, T., Eban-Rothschild, A., Nagari, M., Shemesh, Y., Bloch, G. (2016) Potent social synchronization can override photic entrainment of circadian rhythms. Nat. Commun. 7: 11662.

Fuchikawa, T., Beer, K., Linke-Winnebeck, C., Ben-David, R., Kotowoy, A., Tsang, V. W. K., Warman, G. R., Winnebeck, E. C., Helfrich-Foster, C., Bloch, G. (2017) Neuronal circadian clock protein oscillations are similar in behaviourally rhythmic forager honeybees and in arrhythmic nurses. Open Biol. 7: 170047

George, H., Terracol, R. (1997) The vrille gene of Drosophila is a maternal enhancer of decapentaplegic and encodes a new member of the bZIP family of transcription factors. Genetics 146, 1345-1363.

Gotter A. L., Manganaro T., Weaver D. R., Kolakowski L. F., Possidente B., Sriram S., MacLaughlin D. T., Reppert S. M. (2000) A time-less function for mouse Timeless. Nat. Neurosci. 3: 755-756.

Gu, H., Xiao, J., Niu, L., Wang, B., Ma, G., Dunn, D. W., Huang, D. (2014) Adaptive evolution of the circadian gene timeout in insects. Sci. Rep. 4: 4212

Ingram, K. K., Kutowoi, A., Wurm, Y., Shoemaker, D., Meier, R., Bloch, G. (2012) The Molecular Clockwork of the Fire Ant Solenopsis invicta. PLoS One 7(11): e45715.

Jung, J. H., Seo, Y. H., Seo, P. J., Reyes, J. L., Yun, J., Chua, N. H. and Park, C. M. (2007). The GIGANTEAregulated microRNA172 mediates photoperiodic flowering independent of CONSTANS in Arabidopsis. Plant. Cell. 19, 2736-2748. 
Kadener, S., Stoleru, D., McDonald, M., Nawathean, P., Rosbash, M. (2007). Clockwork Orange is a transcriptional repressor and a new Drosophila circadian pacemaker component. Genes. Dev. 21: 1675-1686.

Kadener, S., Menet, J. S., Sugino, K., Horwich, M. D., Weissbein, U., Nawathean, P., Vagin, V. V., Zamore, P. D., Nelson, S. B. and Rosbash, M. (2009). A role for microRNAs in the Drosophila circadian clock. Genes Dev. 23, 2179-2191.

Kloss, B., Price, J. L., Saez, L., Blau, J., Rothenfluh, A., Wesley, C. S., Young, M. W. (1998). The Drosophila clock gene double-time encodes a protein closely related to human casein kinase I $\varepsilon$. Cell. 94 (1), 97-107.

Knapek, K., Sigrist, S., Tanimoto, H. (2011) Bruchpilot, a synaptic active zone protein for anesthesia-resistant memory. J. Neurosci. 31(9): 3453-3458

Ko, H. W., Jiang, J., \& Edery, I. (2002). Role for Slimb in the degradation of Drosophila Period protein phosphorylated by Doubletime. Nature, 420 (6916), 673678

Kojima, S., Shingle, D. L., \& Green, C. B. (2011). Posttranscriptional control of circadian rhythms. J Cell Sci, 124(3), 311-320.

Kruger, J., Rehmsmeier, M. (2006) RNAhybrid: microRNA target prediction easy, fast and flexible. Nucleic Acids Res. 34: W451-W454.

Lim, C., Chung, B. Y., Pitman, J. L., McGill, J. J., Pradhan, S., Lee, J., Keegan, K. P., Choe, J., Allada, R. (2007) Clockwork orange encodes a transcriptional repressor important for circadian-clock amplitude in Drosophila. Curr Biol 17:1082-1089

Liu, F., Peng, W., Li, Z., Li, W., Li, L., Pan, J., Zhang, S., Miao, Y., Chen, S., Su, S. (2012a) Next-generation small RNA sequencing for microRNAs profiling in Apis mellifera: comparison between nurses and foragers. Insect Mol. Biol. 21: 297-303.

Liu, N., Landreh, M., Cao, K., Abe, M., Hendriks, G. J., Kennerdell, J. R., Zhu, Y., Wang, L. S., Bonini, N. M. (2012b) The microRNA miR-34 modulates ageing and neurodegeneration in Drosophila. Nature. 482 (7386): 519-523.

Livak, K. J., Schmittgen, T. D. (2001) Analysis of relative gene expression data using real-time quantitative PCR and the $2^{-\Delta \Delta C t}$ method. Methods. 25,402-408.

Lourenço, A. P., Mackert, A., Cristino, A. S., Simoes, Z. L. P. (2008) Validation of reference genes for gene expression studies in the honey bee, Apis mellifera, by quantitative real-time RT-PCR. Apidologie. 39, 372385

Luhur, A., Chawla, G., Sokol, N. S. (2013) MicroRNAs as components of systemic signaling pathways in Drosophila melanogaster. Curr. Top. Dev. Biol. 105: 97-123.

Macedo, L. M., Nunes, F. M., Freitas, F. C., Pires, C. V., Tanaka, E. D., Martins, J. R., Piulachs, M. D., Cristino, A. S., Pinheiro, D. G., Simões, Z. L. (2016) MicroRNA signatures characterizing casteindependent ovarian activity in queen and worker honeybees (Apis mellifera L .). Insect Mol Biol. 25: 216226.
Matsumoto, A., Ukai-Tadenuma, M., Yamada, R. G., Houl, J., Umo, K.D., et al. (2007) A functional genomics strategy reveals clockwork orange as a transcriptional regulator in the Drosophila circadian clock. Genes Dev. 21:1687-1700

Meshi, A., Bloch, G. (2007) Monitoring circadian rhythms of individual honey bees in a social environment reveals social influences on postembryonic ontogeny of activity rhythms. J. Biol. Rhythms. 22 (4): 343-355.

Nagari, M., Bloch, G. (2012) The involvement of the antennae in mediating the brood influence on circadian rhythms in "nurse" honey bee (Apis mellifera) workers. J. Insect. Physiol. 58 (8): 1096-1103.

Nandi, A., Vaz, C., Bhattacharya, A., Ramaswamy, R. (2009). miRNA-regulated dynamics in circadian oscillator models. BMC Syst Biol. 3, 45-47.

Panda, S., Hogenesch, J. B., Kay, S. A. (2002) Circadian rhythms from flies to human. Nature 417 (6886): 329335.

Pires, C. V., Freitas, F. C., Cristino, A. S., Dearden, P. K., Simões, Z. L. (2016) Transcriptome Analysis of Honeybee (Apis Mellifera) Haploid and Diploid Embryos Reveals Early Zygotic Transcription during Cleavage. PLoS One 11(1): e0146447.

Price, J. L., Blau, J., Rothenfluh, A., Abodeely, M., Kloss, B., \& Young, M. W. (1998). double-time is a novel Drosophila clock gene that regulates PERIOD protein accumulation. Cell. 94 (1), 83-95.

Qin, Q. H., Wang, Z. L., Tian, L. Q., Gan, H. Y., Zhang, S. W., Zeng, Z. J. (2014). The integrative analysis of microRNA and mRNA expression in Apis mellifera following maze-based visual pattern learning. Insect Sci. 21: 619-36.

Reddy, K. L., Wohlwill, A., Katzen, A., Dzitoeva, S., Lin, M., Holbrook, S., Storti, R.V. (2000) The Drosophila PAR Domain Protein 1 (Pdp1) gene encodes multiple differentially expressed mRNAs and proteins through the use of multiple enhancers and promoters. Dev. Biol. 224, 401-414.

Reddy, K. L., Rovani, M. K., Wohlwill, A., Katzen, A., Storti, R. V. (2006) The Drosophila Par domain protein I gene, $P d p 1$, is a regulator of larval growth, mitosis and endoreplication. Dev. Biol. 289: 100-114

Reischl, S., Kramer, A. (2011). Kinases and phosphatases in the mammalian circadian clock. FEBS Lett. 585 (10), 1393-1399.

Reppert, S. M., Weaver, D. R. (2000) Comparing clockworks: mouse versus fly. J. Biol. Rhythms. 15, 357-64.

Rodriguez-Zas, S. L., Southey, B. R., Shemesh, Y., Rubin, E. B., Cohen, M., Robinson, G. E., Bloch, G. (2012) Microarray Analysis of Natural Socially-Regulated Plasticity in Circadian Rhythms of Honey Bees. J. Biol. Rhythms 27(1): 12-24

Rubin, E. B., Shemesh, Y., Cohen, M., Elgavish, S., Robertson, H. M., Bloch, G. (2006) Molecular and phylogenetic analyses reveal mammalian-like clockwork in the honey bee (Apis mellifera) and shed new light on 
the molecular evolution of the circadian clock. Gen. Res. 16:1352-1365

Sadd, B. M., Barribeau, S. M., Bloch, G., de Graaf, G. C., Dearden, P. (2015) The genomes of two key bumblebee species with primitive eusocial organization. Genome Biol. 16(1): 76.

Sandrelli, F., Costa, R., Kyriacou, C. P., \& Rosato, E. (2008). Comparative analysis of circadian clock genes in insects. Insect. Mol. Biol. 17 (5), 447-46

Sangoram, A. M., Saez, L., Antoch, M. P., Gekakis, N., Staknis, D., et al. (1998) Mammalian circadian autoregulatory loop: a timeless ortholog and mPer1 interact and negatively regulate CLOCK-BMAL1induced transcription. Neuron. 21, 1101-1113

Saunders, D. S. (2002). Insect clocks. Amsterdam: Elsevier.

Shannon, P., Markiel, A., Ozier, O., Baliga, N. S., Wang, J. T., Ramage, D., Amin, N., Schwikowski, B., Ideker, T. (2003) Cytoscape: a software environment for integrated models of biomolecular interaction networks. Genome Res. 13(11): 2498-2504.

Shemesh, Y., Cohen, M., Bloch, G. (2007) Natural plasticity in circadian rhythms is mediated by reorganization in the molecular clockwork in honeybees. FASEB J. 21, 2304-2311.

Shemesh, Y., Eban-Rothschild, A., Cohen, M., Bloch, G. (2010) Molecular dynamics and social regulation of context-dependent plasticity in the circadian clockwork of the honey bee. J. Neurosci. 30, 12517-25.

Sire, C., Moreno, A. B., Garcia-Chapa, M., Lopez-Moya, J. J., San Segundo, B. (2009). Diurnal oscillation in the accumulation of Arabidopsis microRNAs, miR167, miR168, miR171 and miR398. FEBS Lett. 583, 1039-1044.

Szuplewski, S., Kottler, B., \& Terracol, R. (2003). The Drosophila bZIP transcription factor Vrille is involved in hair and cell growth. Development. 130 (16), 36513662.

Szuplewski, S., Fraisse-Véron, I., George, H., Terracol, R. (2010) vrille is required to ensure tracheal integrity in Drosophila embryo. Dev. Growth Differ. 52 (5): 409418.

Takumi, T., Nagamine, Y., Miyake, S., Matsubara, C., Taguchi, K., et al. (1999) A mammalian ortholog of Drosophila timeless, highly expressed in SCN and retina, forms a complex with mPER1. Genes Cells. 4, 67-75.

Toma, D. P., Bloch, G., Moore, D., Robinson, G. E. (2000) Changes in period mRNA levels in the brain and division of labor in honey bee colonies. Proc. Nat. Acad. Sci. U S A. 97:6914-6919.
Tomioka, K., Matsumoto, A. (2010) A comparative view of insect circadian clock systems. Cell. Mol. Life Sci. 67:1397-1406.

Vansteensel, M. J., Michel, S., Meijer, J. H. (2008) Organization of cell and tissue circadian pacemakers: A comparison among species. Brain Res. Rev. 58 (1): $18-47$

Wagh, D. A., Rasse, T. M., Asan, E., Hofbauer, A., Schwenkert, I., et al. (2006) Bruchpilot, a protein with homology to ELKS/CAST, is required for structural integrity and function of synaptic active zones in Drosophila. Neuron. 49(6): 833-844.

Weaver, D. B., Anzola, J. M., Evans, J. D., Reid, J. G., Reese, J. T., Childs, K. L., Zdobnov, E. M., Samanta, M. P., Miller, J., Elsik, C. G. (2007) Computational and transcriptional evidence for microRNAs in the honey bee genome. Genome Biol. 8 (6): R97.

Weber, F., Zorn, D., Rademacher, C., \& Hung, H. C. (2011). Post-translational timing mechanisms of the Drosophila circadian clock. FEBS Lett. 585 (10), 1443-1449

Weinstock, G. M., Robinson, G. E., Gibbs, R. A., Worley, K. C., Evans, J. D. et al. (2006) Insights into social insects from the genome of the honeybee Apis mellifera. Nature, 443 (7114), 931-949.

Woźnicka, O., Görlich, A., Sigrist, S., Pyza, E. (2015) BRP-170 and BRP190 isoforms of Bruchpilot protein differentially contribute to the frequency of synapses and synaptic circadian plasticity in the visual system of Drosophila. Front. Cell. Neurosc. 9: 238

Xing, W., Busino, L., Hinds, T. R., Marionni, S. T., Saifee, N. H., Bush, M. F., .. \& Zheng, N. (2013). SCFFBXL3 ubiquitin ligase targets cryptochromes at their cofactor pocket. Nature, 496 (7443), 64-68

Xu, S., Witmer, P. D., Lumayag, S., Kovacs, B., Valle, D. (2007). MicroRNA (miRNA) transcriptome of mouse retina and identification of a sensory organ-specific miRNA cluster. J. Biol. Chem. 282, 25053-25066.

Yang, M., Lee, Jung-Eun., Padgett, R. W., Edery, I. (2008) Circadian regulation of a limited set of conserved microRNAs in Drosophila. BMC Genomics, 9:83.

Yoo, S. H., Mohawk, J. A., Siepka, S. M., Shan, Y., Huh, S. K., Hong, H. K., ... \& Nussbaum, J. (2013). Competing E3 ubiquitin ligases govern circadian periodicity by degradation of CRY in nucleus and cytoplasm. Cell, 152 (5), 1091-1105.

Young, M. W., Kay, S. A. (2001) Time zones: A comparative genetics of circadian clocks. Nat. Rev. Genet. 2 (9): 702-715 